\title{
The Concept of Economic Evaluation and Its Application in Thyroid Cancer Research
}

\author{
Kyungsik Kim ${ }^{1,2,3}$, Mijin Kim ${ }^{4, *}$, Woojin Lim ${ }^{1,2,3}$, Bo Hyun Kim4, Sue K. Park ${ }^{1,3,5}$ \\ ${ }^{1}$ Department of Preventive Medicine, Seoul National University College of Medicine; ${ }^{2}$ Department of Biomedical Sciences, \\ Seoul National University Graduate School; ${ }^{3}$ Cancer Research Institute, Seoul National University, Seoul; ${ }^{4}$ Department of \\ Internal Medicine, Biomedical Research Institute, Pusan National University Hospital, Busan; ${ }^{5}$ Integrated Major in Innovative \\ Medical Science, Seoul National University College of Medicine, Seoul, Korea
}

Economic evaluation is a type of comparative analysis between interventions in terms of both their resource use and health outcomes. Due to the good prognosis of thyroid cancer (TC), the socioeconomic burden of TC patients post-diagnosis is increasing. Therefore, economic evaluation studies focusing on TC are recommended. This study aimed to describe the concept and methods of economic evaluation and reviewed previous TC studies. Several previous studies compared the costs of interventions or evaluated recurrence, complications, or quality of life as measures of their effectiveness. Regarding costs, most studies focused on direct costs and applied hypothetical models. Cost-minimization analysis should be distinguished from simple cost analysis. Furthermore, due to the universality of the term "cost-effectiveness analysis" (CEA), several studies have not distinguished CEA from cost-utility analysis; this point needs to be considered in future research. Cost-benefit analyses have not been conducted in previous TC research. Since TC has a high survival rate and good prognosis, the need for economic evaluations has recently been pointed out. Therefore, correct concepts and methods are needed to obtain clear economic evaluation results. On this basis, it will be possible to provide appropriate guidelines for TC treatment and management in the future.

Keywords: Thyroid neoplasms; Economic evaluation; Cost; Effectiveness; Cost-effectiveness analysis

\section{INTRODUCTION}

The incidence of thyroid cancer has markedly increased worldwide in recent decades [1]. In South Korea (hereafter, Korea), the incidence rate of thyroid cancer increased sharply by more than 10 times between 2000 and 2012 (age-standardized inci-

Received: 22 June 2021, Revised: 26 July 2021, Accepted: 28 July 2021

Corresponding authors: Bo Hyun Kim

Department of Internal Medicine, Biomedical Research Institute, Pusan National University Hospital, 305 Gudeok-ro, Seo-gu, Busan 49241, Korea

Tel: +82-51-240-7236, Fax: +82-51-254-3237, E-mail: pons71@hanmail.net

Sue K. Park

Department of Preventive Medicine, Seoul National University College of

Medicine, 103 Daehak-ro, Jongno-gu, Seoul 03080, Korea

Tel: +82-2-740-8338, Fax: +82-2-747-4830, E-mail: suepark@snu.ac.kr dence rate [ASIR] based on the Korean population in 2000: 7.2 per 100,000 in 2000; 74.8 per 100,000 in 2012), decreased from 2012 to 2015, and then slightly increased again after 2015 [2]. Although the incidence of thyroid cancer in Korea has changed to a pattern of a slow increase since 2015, the incidence in 2018 is still the highest in the world, twice that of the United States

Copyright $(2021$ Korean Endocrine Society

This is an Open Access article distributed under the terms of the Creative Commons Attribution Non-Commercial License (https://creativecommons.org/ licenses/by-nc/4.0/) which permits unrestricted non-commercial use, distribution, and reproduction in any medium, provided the original work is properly cited.

*These authors contributed equally to this work. 
and three times that of Canada [3].

Thyroid cancer has a 5-year survival rate of almost 100\%, irrespective of whether surgery is performed [2]. Furthermore, it has been suggested that the rapid increase of the ASIR of thyroid cancer in Korea may reflect over-diagnosis and overscreening [4]. Collectively, these points call into question the value of thyroid cancer screening. However, there are limitations in making an appropriate choice because no comparative results have been reported. It is also necessary to select appropriate medical services in a resource-limited environment. Economic evaluation methodologies are useful for resolving such issues [5].

With its system of universal coverage through the National Health Insurance Service, Korea is facing a variety of issues, such as providing adequate services to the public, guaranteeing people's right to make choices for their own health, and ensuring efficient compensation for providers [6]. Since thyroid cancer is a disease with a favorable prognosis, the problems in Korea also encompass challenges in screening, diagnosis, treatment, and long-term health care for thyroid cancer, suggesting that an appropriate allocation of limited resources is necessary. Thus, economic evaluation methodology is particularly important because efficient reimbursement in the National Health Insurance Service and people's right to make choices for their own health are both important issues. Therefore, it is vitally important for thyroid cancer research to understand and apply economic evaluation methodology.

The purpose of this study was to introduce the methods of economic evaluation, key elements, and key indicators. To present economic evaluation methodology in an easy-to-understand way for thyroid cancer researchers, we briefly introduce several representative examples of previous thyroid cancer studies using economic evaluation methods. We also present several applicable examples from studies currently in progress at the Korean Thyroid Association.

\section{METHODS}

This review focused on illustrating the concept and methods of economic evaluation with examples from thyroid cancer research. First, in order to explain the concept and methods of economic evaluation in an easy-to-understand way for thyroid cancer researchers, textbooks on economic evaluation were selected and summarized based on keywords and main methods [7,8]. Existing thyroid cancer studies applying the economic evaluation methodology were searched up to May 31, 2021 using a combination of keywords: ("Thyroid Cancer" \& ["Cost analysis", or "Cost-minimization analysis" or "Cost-effectiveness analysis" or "Cost-utility analysis"]) in MEDLINE (PubMed) and Google Scholar. A search was also performed with the same keywords in the Cochrane Database. The resulting 1,323 papers were all searched using the keywords, and after excluding irrelevant papers such as inappropriate literature based on the abstracts and titles, non-English papers, and papers with no access to the full text, 49 papers remained. Among them, we selected examples of straightforward interventions that are easy to understand for thyroid cancer researchers. Four thyroid cancer researchers selected each topic, and one to two examples of each method were selected through discussion and consensus.

\section{RESULTS}

\section{Concepts and main methods of economic evaluation}

Economic evaluation is a type of comparative analysis between health interventions in terms of both their resource use (costs) and health outcomes $[9,10]$. Its goal is usually to allocate resources with sufficient efficiency that the maximum outcomes can be expected in a complicated health care environment $[9,10]$.

Based on the methods and outcomes, economic evaluations can be classified into four types: cost-minimization analysis (CMA), cost-effectiveness analysis (CEA), cost-utility analysis (CUA), or cost-benefit analysis (CBA) (Table 1) [11]. In these types of economic evaluations, costs are measured in the same way, but the outcomes (cost, effectiveness, utility, and benefit) and their measurement methods are different.

CMA is a method of selecting a more economical intervention by comparing the cost of the interventions when the clinical effects (e.g., in terms of therapeutic effects between generics and patent medicines, safety and tolerability, and quality of life) of each intervention is the same as a prerequisite [12]. It calculates the additional cost of the intervention compared to the comparative intervention for one thyroid cancer case. An assumption of this approach is that the intervention and the comparative intervention have the same effect. A positive number indicates additional cost and a negative number indicates reduced cost. CMA has the benefit of simple and easy interpretation, and it is commonly used to compare generics and patent medicines as standard medications. However, CMA is inappropriate when there are differences in the effects of the intervention and comparison or in the measures and units of the effects.

If the clinical outcome and input cost of the targeted interven- 
Table 1. Basic Concepts of Economic Evaluation

\begin{tabular}{|c|c|c|c|}
\hline Method & Description & Measures & Indicators \\
\hline $\begin{array}{l}\text { Cost-minimization } \\
\text { analysis (CMA) }\end{array}$ & $\begin{array}{l}\text { Comparison of costs when the intervention } \\
\text { group and comparison group have clearly } \\
\text { equivalent clinical effects }\end{array}$ & $\begin{array}{l}\text { Only the cost is measured; the effects are not measured } \\
\text { since these are assumed to be equal. }\end{array}$ & $\begin{array}{l}\text { Excess or } \\
\text { additional } \\
\text { cost per case }\end{array}$ \\
\hline $\begin{array}{l}\text { Cost-effectiveness } \\
\text { analysis (CEA) }\end{array}$ & $\begin{array}{l}\text { Comparison of the relative ratio of cost and } \\
\text { effectiveness between an intervention group } \\
\text { and a comparison group, which are estimated } \\
\text { as different values }\end{array}$ & $\begin{array}{l}\text { This method calculates the cost difference (numerator) of } \\
\text { the two interventions per the difference in outcomes } \\
\text { (denominator) of the two interventions as the ICER. The } \\
\text { ICER indicates the incremental (decreased) cost per } 1 \\
\text { unit of increased effectiveness. }\end{array}$ & ICER \\
\hline $\begin{array}{l}\text { Cost-utility analysis } \\
\text { (CUA) }\end{array}$ & $\begin{array}{l}\text { Comparison of different diseases or projects } \\
\text { with different units of measure, considering } \\
\text { quality of life (health status) }\end{array}$ & $\begin{array}{l}\text { This method calculates the cost per 1-unit increment of } \\
\text { the effect on the health status as the ICUR. A life-year at } \\
\text { perfect health status is considered as } 1 \text { QALY and a } \\
\text { life-year at death is 0 QALY. }\end{array}$ & ICUR \\
\hline $\begin{array}{l}\text { Cost-benefit analysis } \\
\text { (CBA) }\end{array}$ & $\begin{array}{l}\text { Method of converting all possible alternatives } \\
\text { into monetary values and comparing the } \\
\text { benefits per cost (in monetary terms) of the } \\
\text { alternatives }\end{array}$ & Monetary units & BCR \\
\hline
\end{tabular}

ICER, incremental cost-effectiveness ratio; ICUR, incremental cost-utility ratio; QALY, quality-adjusted life-year; BCR; benefit-cost ratio.

tion and the comparative intervention are different, we calculate the cost difference (numerator) of the two interventions per the difference in outcomes (denominator) of the two interventions, which is the additional cost of the targeted intervention per a 1-unit increment of the outcome. Here, the result of each intervention is clinical effectiveness for CEA and quality of life for the CUA. The incremental cost-effectiveness ratio (ICER), which identifies the incremental (decreased) cost per 1-unit increased effectiveness, is used in the CEA framework [13]. CEA is the main decision-making tool in economic evaluations, and the effectiveness of interventions is measured in "natural units," such as the recurrence rate, complication-free survival, complication incidence, or life-years [7]. For decision-making, it is necessary to compare the estimated ICER with the maximum acceptable ICER, which represents the maximum amount that society is willing to pay for an additional unit of health outcome [14]. The main advantage of CEA is that measuring outcomes in natural units simplifies the analysis, making it intuitive and facilitating easier communication among physicians. However, CEA faces limitations when comparing different diseases or projects with different measurement units. As another limitation, CEA overlooks quality of life; therefore, CUA has been suggested as an alternative method for analysis [8]. Similar to CEA, CUA uses the incremental cost-utility ratio (ICUR) as the outcome. The ICUR is defined as the incremental (decreased) cost per 1-unit of increased utility (quality of life or quality-adjusted life-year [QALY] due to thyroid cancer treatment or com- plications). Additionally, some previous studies dealt with quality of life or QALY from cancer treatment and complications as measures of utility [15-17].

CBA is a method of converting all possible alternatives into monetary values and comparing the benefits per cost (in monetary terms) of the alternatives. CBA compares the benefits of two alternatives according to their costs. To choose between alternatives, the costs should be assessed for both alternatives. If the costs of both alternatives are the same, the alternative with the larger benefit would be selected [18]. It is a method of measuring the opportunity cost of investing resources for their efficient allocation. CBA estimates the benefit per cost of a specific intervention, reported as the benefit-cost ratio (BCR). A BCR is greater than 1 indicates that the net present value of the project exceeds zero [19]. The BCR is commonly used in commercial transactions, business or policy decisions, and project investments [20]. When evaluating the economic value of interventions for medical health services, CBA is rarely used because it is difficult to assign a monetary value to clinical results [19]. Therefore, in the latter context, it is recommended to use CEA or CUA.

Of particular note, in economic evaluation, the main economic evaluation method should be selected before constructing the analysis model. The model can be a decision tree or Markov model (Supplemental Fig. S1). A decision tree model consists of decision nodes (squares), chance nodes (circles), probabilities, and outcome nodes (triangles), as shown in Supplemental Fig. 
S1A. A decision node is defined as a point where the decisionmaker makes a choice. A chance node is defined as a probabilistic event beyond the control of the decision-maker. In addition, each chance node has probability branches, the sum of which should be 1 . The probability can be estimated from actual data or a literature review. A Markov model is conducted based on the health status of a study population during an observation period. It has a constant rate of health transition for each health status over time, and it accumulates the cost or quality of life while individuals stay in that health status. The health statuses used in a Markov model should be mutually exclusive, and the model should have as simple a design as possible. Additionally, as shown in Supplemental Fig. S1B, there is a probability of moving to and from each state, which is defined as the transition probability. The sum of the transition probabilities for moving from each health status to other statuses should also be 1 (e.g., probability of moving from mild status to mild status, 0.37 ; from mild to moderate, 0.49 ; from mild to severe, 0.11 ; and from mild to death, $0.03 ; 0.37+0.49+0.11+0.03=1$ ).

\section{Costs and outcomes as key elements in economic evaluation Costs}

One of the main challenges in economic evaluation analyses is to decide which costs should be included and how these costs should be measured and valued. Costs can be divided into direct, indirect, and intangible costs (Fig. 1) [21]. Direct costs can be divided into medical and non-medical costs. Direct medical costs are further divided into fixed costs, which must be paid irrespective of treatment (e.g., facility management and building leases), and variable costs, which vary depending on the amount of health care resources used (e.g., inpatient, outpatient, and pharmaceutical costs). Non-medical costs encompass transportation for outpatient, inpatient, and social services [22]. Because of individuals' personal interest in their health status, it is also necessary to consider health supplements and alternative medicine within the category of complementary and alternative medicine (CAM) [23]. Although CAM is not included as a medical cost, it is relevant for a detailed accounting of non-medical costs. Indirect costs include loss of productivity, the value of the output lost due to a patient's death (converted to monetary value), and inpatient time loss due to a patient's morbidity or injury. Intangible costs are those incurred for psychological damages such as a patient's anxiety and pain (converted to monetary value) [22]. Intangible costs are treated as a type of outcome in CEA and CUA, but as costs in CBA.

The value of current costs and future costs cannot be compared equally. Due to the time preference for the present, the value of future costs (the future monetary value) is lower than that of current costs (the current monetary value) [24]. The discount rate is used to correct for the future value. If the discount rate is 3\% and considered 5-year of period the following formula can be applied: [present value] $=[$ future value $] \times 1 /(1+0.03)^{5}$. The discount rate is applied in the process of assessing costs and benefits at different times. The discount rate varies depending on the economic status of each country and its health care system, and it should be considered in economic evaluations.

\section{Outcomes}

Outcome indicators vary depending on the types of economic evaluation. They can be divided into (1) clinical outcomes, (2) psychosocial outcomes, and (3) economic outcomes. Clinical outcomes consist of complication-free survival, morbidity, mor-

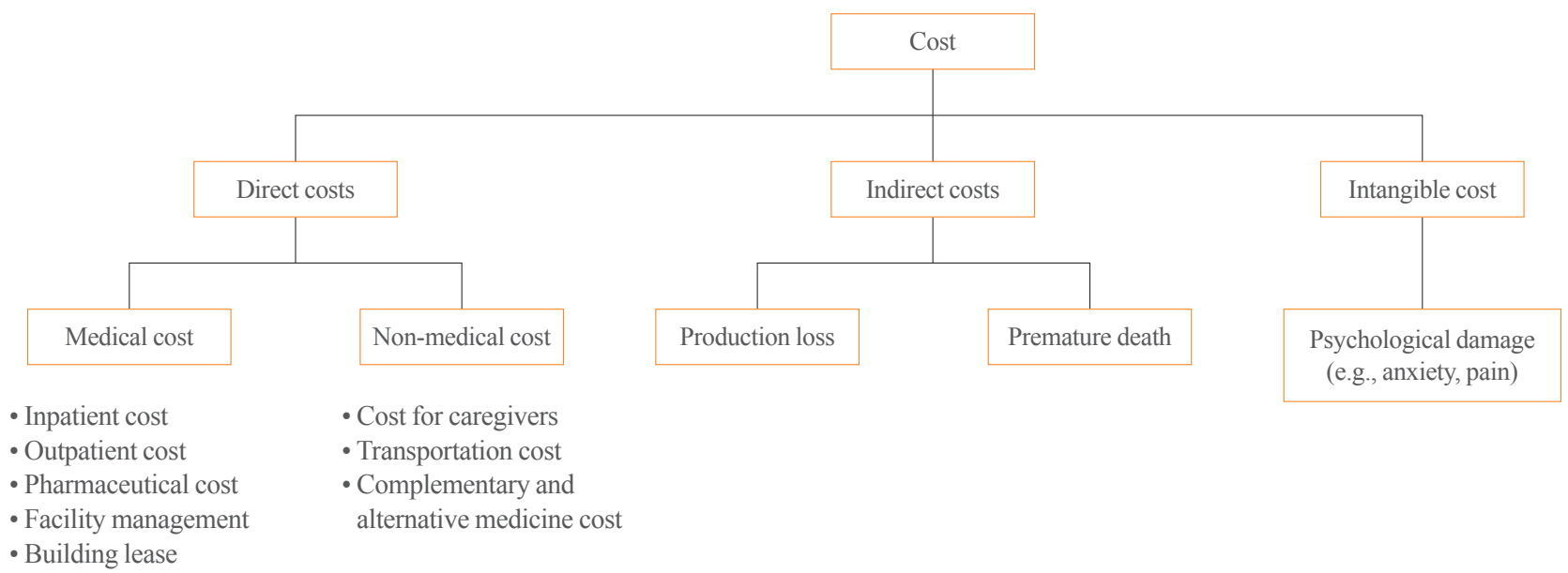

Fig. 1. Main types of costs in economic evaluations. 
tality, or complications resulting from interventions for thyroid cancer recurrence. Psychosocial outcomes consist of utilitybased quality of life metrics (e.g., QALY). In particular, QALY is a generic measure of the outcome of medical interventions, including both the quality and the quantity of life [25]. The duration of time an individual is likely to spend in a health condition is weighted by a utility score from standard valuations (Fig. 2 ). Health-related quality of life is generally expressed on a numerical scale ranging from 0 (death) to 1 (perfect health), and it assumes that a year lived in perfect health is worth 1 QALY [25]. QALY is usually used for CUA. Lastly, economic outcomes include productivity loss, such as absence from work and work changes.

In CEA, clinical outcomes are mainly used as indicators of effectiveness, whereas in CUA, psychosocial results that have been adjusted for quality of life are mainly used as indicators of utility. Economic outcomes are generally included as indirect costs when estimating costs. In CBA, the benefit of an investment is estimated by considering the increase or decrease of all the above-mentioned results after the implementation of a specific program.

\section{Examples of economic evaluation in previous thyroid cancer studies \\ Cost minimization analysis}

A previous study conducted in 2014 in Hong Kong sought to determine whether total thyroidectomy or total thyroidectomy with prophylactic central neck dissection was more cost-saving for nodal-negative (cN0) papillary thyroid cancer (PTC) (Table 2) [26]. A hypothetical model was applied based on a decision tree design for 20 years. The reference case was a non-pregnant fe-

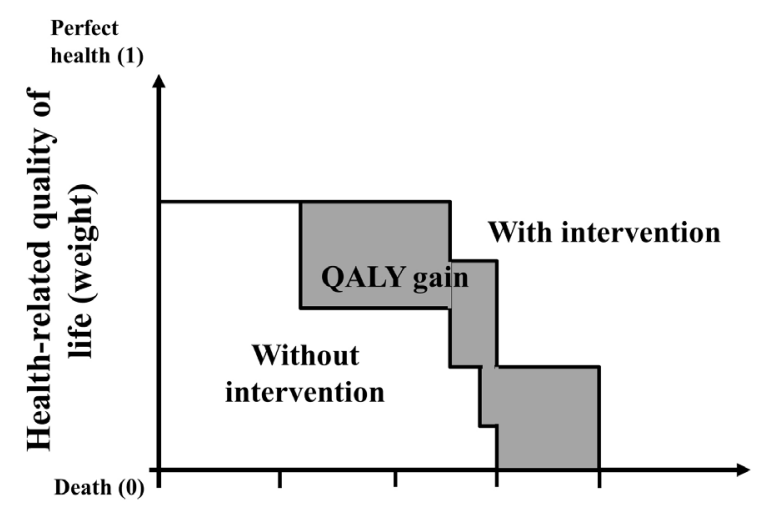

\section{Survival (follow-up) years}

Fig. 2. Basic principle of the quality-adjusted life year (QALY) in economic evaluations. male patient aged 50 years with a unifocal intrathyroidal $1.5-\mathrm{cm}$ cN0 PTC. For cost estimation, the study applied an institutional perspective, considering only direct costs. For medical services provided within the national or state health care system, direct cost information was estimated from the government website or its publications. As cost items, workup procedures, complications, hospitalizations, and drugs related to the operation were included and derived from the government database. The probability of death based on reoperation, complications, or non-thyroidal causes following initial surgery was estimated from the literature. A 3\% discount rate was applied for cumulative cost calculation, a sensitivity analysis was conducted, and the increased cost per patient was presented as economic evaluation indicators. The CMA analysis suggested that total thyroidectomy alone is less costly than total thyroidectomy with prophylactic central neck dissection in nodal-negative PTC (\$6,702.81 vs. $\$ 10,062.35)$, with a consistent finding also reported for 20 -year cumulative costs $(\$ 19,888.36$ vs. $\$ 22,760.86)$.

\section{Cost-effectiveness analysis}

Many previous economic evaluation studies compared interventions to assess which management strategy (e.g., different surgery types) is more appropriate in thyroid cancer patients (Table 2) $[27,28]$. The two studies selected as examples of CEA compared two thyroid cancer surgical methods by calculating the additional costs per 1-unit increment of the clinical effectiveness of each surgical method relative to each other [27,28]. In a 2007 study, surgical complications, cause-specific mortality, the survival rate, and the recurrence rate were defined as effectiveness metrics for hemi-thyroidectomy and total thyroidectomy (Table 2) [27]. To assess the effectiveness of each intervention, a Markov model was constructed with a 20 -year follow-up period. The probability of effectiveness according to the intervention was derived from the literature. For cost estimation, that study applied direct costs, such as diagnostic examinations including ultrasonography or fine-needle aspiration, surgery, and non-surgical treatment based on the New York state government and Medicare cost databases. The value of death resulting from unintentional impairment was also considered. The discount rate was defined as $6 \%$, and the sensitivity analysis suggested acceptable thresholds for various parameters. The researchers concluded that total thyroidectomy was cost-effective compared to hemi-thyroidectomy based on the ICER. Total thyroidectomy was predominantly cost-effective based on overall survival (ICER: total thyroidectomy, \$13,979; hemi-thyroidectomy, $\$ 15,184$ ), recurrence-free survival (ICER: total thyroidectomy, 


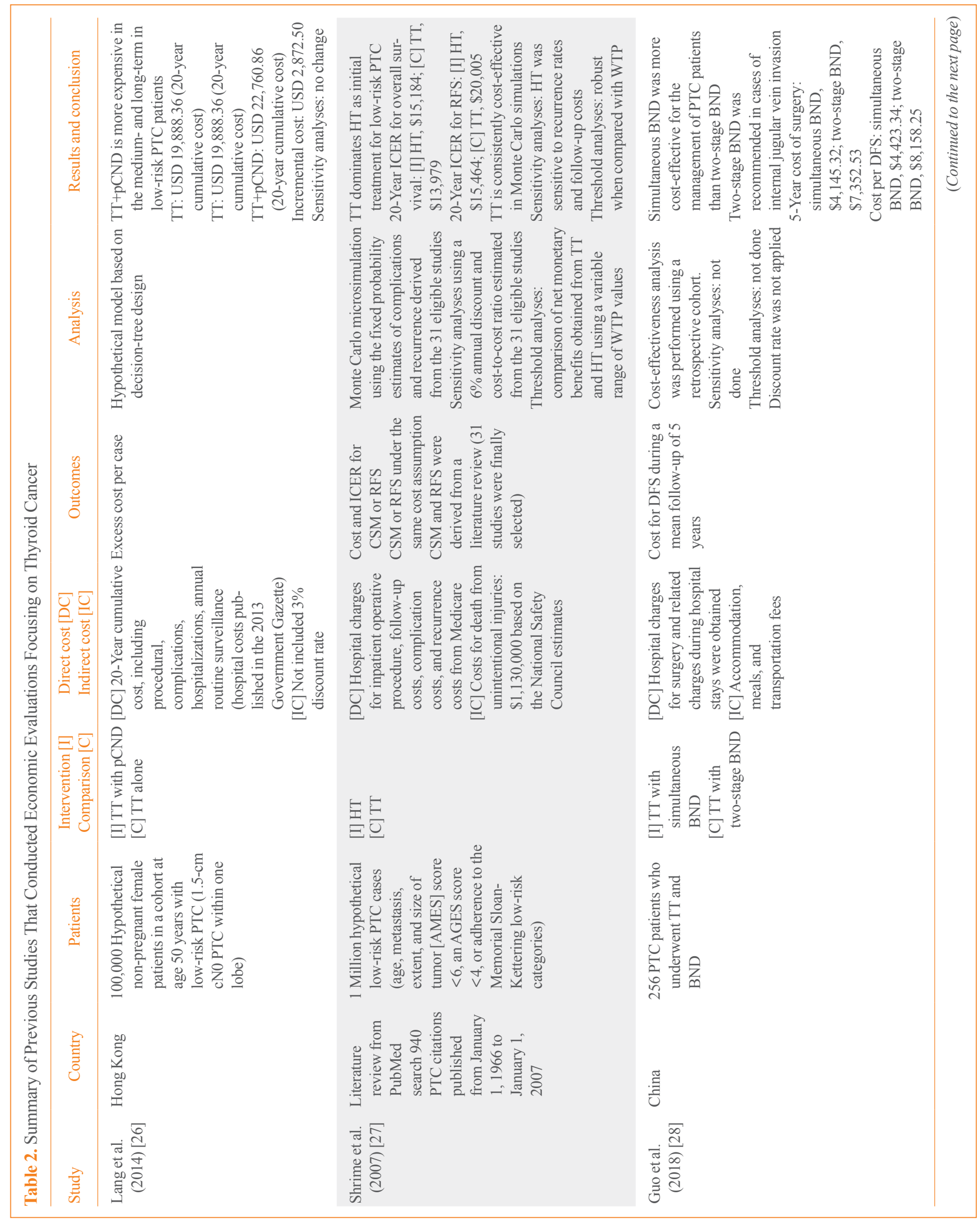




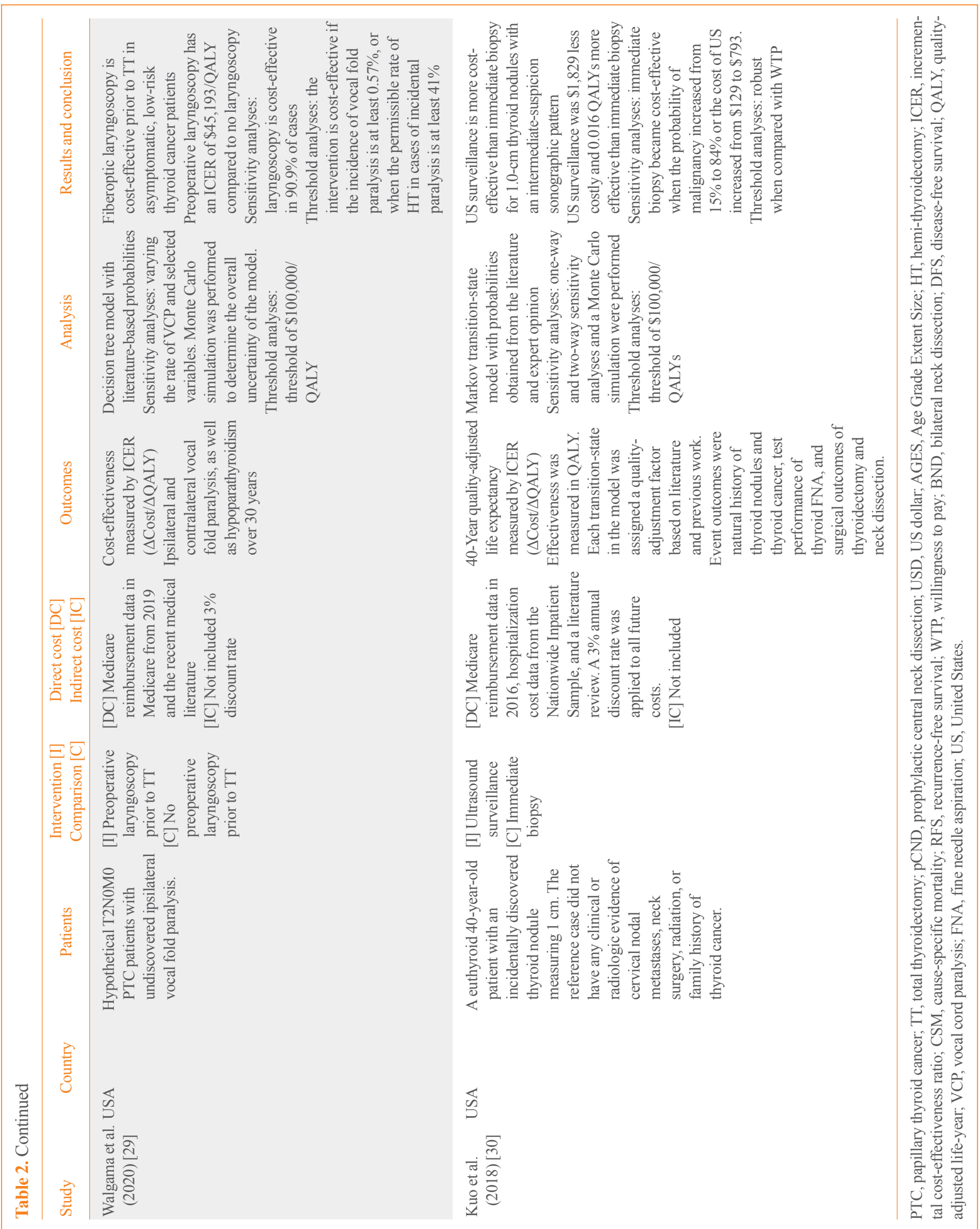


$\$ 15,464$; hemi-thyroidectomy, $\$ 20,005$ ) compared to hemi-thyroidectomy. However, it showed heterogeneity according to the institution in long-term treatment outcomes.

Another study focused on two intervention strategies for bilateral neck dissection (BND): simultaneous and two-stage operations (Table 2) [28]. Patients with bilateral PTC from 2006 to 2012 who did not have a prior history of surgery or radiation exposure comprised the study population. Their pathology records were identified from postoperative clinical data. Surgical and medical expenses including neck dissection, ultrasonography, computed tomography, fine-needle aspiration, and outpatient, inpatient, and postoperative treatment charged by the hospital were considered as medical expenses. As indirect costs, accommodation, meals, and transportation fees were included. Disease-free status was defined as serum thyroglobulin levels of $1 \mathrm{ng} / \mathrm{mL}$ after recombinant human thyroid-stimulating hormone (TSH) therapy, negative neck ultrasound findings, and undetectable thyroglobulin antibody. A discount rate was not considered, and sensitivity and threshold analyses were not performed. The simultaneous BND strategy (cost-effectiveness ratio, defined as the cost per disease-free survivor: $\$ 4,423.34$ ) was more cost-effective than the two-stage BND strategy (cost-effectiveness ratio: $\$ 8,158.25$ ). However, two-stage BND was recommended in cases with invasion of the internal jugular vein.

\section{Cost-utility analysis}

CUA is a type of CEA used to compare the cost of a target intervention and a comparative intervention for thyroid cancer and the effect on the health status of patients who receive the intervention to determine the cost per 1-unit increment of the effect on health status. A life-year at perfect health status (theoretical life-year) is considered as 1 QALY and a life-year at death is 0 QALY [25]. In thyroid cancer, the meaning of "perfect health" is the status of no complications or no recurrence after primary treatment of thyroid cancer or having the best quality of life after maintenance treatment. The former two scales can estimate QALY as a value distributed between 1 QALY in the optimal state and 0 QALY in the death state using complications or recurrence-free years in the population. When using a health-related quality of life questionnaire such as the 36-item ShortForm Health Survey, the optimal quality of life is 1 QALY and the worst quality of life is 0 QALY $[7,8]$. QALY values between 0 and 1 can be estimated through the distribution of scores in each questionnaire and the validation of weights in a randomly selected sample population [7,8,25]. However, the conversion method depends on whether QALY is measured as a value at a single point in time or a cumulative value over several years; therefore, it is necessary to clarify how the results will be operationalized in advance.

A study published in 2020 conducted CUA for fiberoptic laryngoscopy prior to total thyroidectomy in low-risk thyroid cancer patients (T2N0M0 PTC) based on a decision model for 30 years (Table 2) [29]. The study only considered direct costs, which were converted into 2019 direct costs according to public expense data (Medicare) and the literature with a 3\% discount rate. The probabilities and health utility values (ipsilateral and contralateral vocal fold paralysis, as well as hypoparathyroidism) in the model were derived from the literature including complication-free survival years and recurrence-free survival years according to the intervention. The threshold for willingness-to-pay was defined as 100,000(\$)/QALY. Given the sensitivity analysis, cost-acceptability curve, and time horizon according to each parameter in CUA, fiberoptic laryngoscopy prior to total thyroidectomy was found to be cost-effective for lowrisk thyroid cancer patients (\$45,193/QALY) and acceptable based on the willingness-to-pay threshold. As a sensitivity analysis, laryngoscopy was found to be cost-effective in $90.9 \%$ of cases, and the intervention was cost-effective if the incidence of vocal fold paralysis was at least $0.57 \%$.

Another topic of interest is the immediate biopsy and active surveillance of thyroid nodules at 1-cm levels (Table 2) [30]. Therefore, a Markov model was constructed for CUA between immediate biopsy and active surveillance, and the reference case was a 40-year-old woman with 1-cm nodules in 2018. As inclusion criteria, the study considered patients without clinical or imaging evidence of cervical nodal metastases, a history of radiation exposure, or a family history of thyroid cancer. The outcome was defined as the performance and surgical outcomes of fine-needle aspiration, and the probability was derived from the literature. If the probability could not be identified from the literature, it was replaced by expert opinion. The effectiveness of each intervention was measured in QALYs. The time period was 50 years, but the interval of the ultrasound examinations was specifically set according to the nodule condition. For costs, medical and hospitalization fees were considered from the Medicare database or obtained from the literature if they could not be identified from the database, and a 3\% discount rate was applied. The threshold value for willingness-to-pay was defined as $\$ 100,000 / \mathrm{QALY}$. Through sensitivity analyses, the dominance point between active surveillance and biopsy was identified, as was the change in ICUR according to the malignancy probability. As a conclusion, active surveillance with ultraso- 
nography was more cost-effective than an immediate biopsy (incremental cost, \$1,829; QALY, 0.016), with consistent dominance when the probability of malignancy is less than $84 \%$ and if the cost of ultrasonography does not exceed \$793. However, if active surveillance caused considerable anxiety, immediate biopsy was more effective; therefore, quality of life should be considered for each intervention.

\section{Cost-benefit analysis}

CBA is generally used as a preliminary feasibility assessment of financial projects or as an analysis comparing the cost-benefit ratio between interventions. For example, rapid-transit railway enterprise and new town developments are domains where CBA has been widely performed. A single intervention can be judged as an economical project if the BCR is greater than 1 or if the net benefit is greater than 0 . However, no previous CBA studies have been conducted on thyroid cancer.

\section{DISCUSSION}

In this study, the concept and methods of economic evaluation were summarized briefly with a focus on thyroid cancer, and we intended to help readers understand economic evaluation based on examples from previous studies. To conduct an economic evaluation for thyroid cancer, the definitions of several key elements should be discussed and clarified between researchers.

The first points to consider are direct, indirect, and intangible costs [21]. Most previous studies used direct costs, but some studies also used complication costs, the value of death, and indirect costs [26-30]. Previous thyroid cancer studies used discount rates of $3 \%$ to $6 \%$, although there were variations across countries and research periods. Costs were most commonly obtained from public expenditure databases such as Medicare or the previous literature, and the probabilities of outcomes according to interventions were derived from previous studies [26-30]. For the economic evaluation analyses, decision tree or Markov models were applied as hypothetical models.

The second point to consider is the outcome. In CMA, it is common to present an excess cost per case or patient, assuming the same effect. However, most previous studies compared costs between interventions, which requires a clear distinction [3136]. Because of the universality of the term "CEA," regardless of the study type, numerous CUA studies have been reported as CEA studies, and the indicators were also presented as the ICER, not the ICUR [37-41]. It is possible to evaluate whether the derived ICER or ICUR is socially acceptable compared to thresholds for decision-making, as social acceptability defines the concept of willingness-to-pay. Socially acceptable marginal costs vary from country to country and over time. In studies in the United States, the cost per QALY is considered socially acceptable if it is lower than per capita gross domestic product (GDP). In 2008, the United States per capita GDP was about 48,000 dollars, and the United States threshold was therefore $\$ 50,000 /$ QALY [42].

A highlight of recent research on thyroid cancer is the economic evaluation of surgery and active surveillance in patients with low-risk thyroid cancer [33,34,40,43]. Due to the high survival rate and good prognosis of low-risk thyroid cancer, especially papillary thyroid microcarcinoma (PTMC), the American Thyroid Association recommended that immediate surgery is not necessary for patients with low-risk tumors such as PTMC without clinically evident metastases or local invasion [44]. However, active surveillance using ultrasonography also leads to an economic burden in the long term, and patients may also live with the anxiety that cancer might develop. These points need to be considered in an economic evaluation. In this context, a prospective cohort for PTMC patients in Korea (Multicenter Prospective Cohort Study of Active Surveillance on Papillary Thyroid Microcarcinoma [MAeSTro]) was conducted to compare lobectomy and active surveillance in 2016 [45]. MAeSTro is a pragmatic cohort study with the aim of evaluating the evidence regarding whether active surveillance is helpful in reducing unnecessary surgery in patients with small, low-risk thyroid cancer. Thyroid cancer recurrence, complications, and quality of life were compared as measures of effectiveness between the two interventions. Furthermore, researchers at the Korean Thyroid Association launched a practical clinical trial (the Multicenter Randomized Controlled Study for Assessing the Usefulness of Suppressing Thyroid Stimulating Hormone Target Levels after Thyroid lobEctomy in Low to Intermediate Risk Thyroid Cancer Patients [MASTER] study) for thyroid cancer patients who underwent lobectomy for low-risk and intermediate-risk cancer to compare recurrence, quality of life, and complications (including cardiovascular disease, osteoporosis, and metabolic disease, and musculoskeletal disease) [46]. For the first study, if the effects of the two interventions are the same, the economic benefit can be evaluated through CMA. The second study aims to establish evidence about how long TSH levels should be maintained in TSH suppression therapy. The degree of levothyroxine use differs according to the maintenance of TSH levels; thus, TSH suppression therapy has both clinical benefits and risks. Therefore, in addition to CEA, an 
evaluation through CUA considering quality of life should be performed.

\section{CONCLUSIONS}

In this study, we described the concept and methods of economic evaluation and reviewed previous studies focusing on thyroid cancer. Thyroid cancer has a favorable prognosis, with a high chance of long-term survival. Because of the characteristics of thyroid cancer, it is controversial whether the medical costs administered for thyroid cancer are appropriate. In particular, in countries with a national health insurance system such as Korea, it is necessary to select the most efficient and optimal method to support various medical services in a balanced way within limited financial resources. Economic evaluation is a method for making optimally cost-efficient choices in a resource-limited setting. By understanding and applying the precise concepts and methods of economic evaluation, we can produce comparative results to elucidate controversial issues in an evidence-based manner. Economic evaluation studies are expected to provide appropriate guidelines for thyroid cancer surveillance, diagnosis, and management.

\section{CONFLICTS OF INTEREST}

No potential conflict of interest relevant to this article was reported.

\section{ACKNOWLEDGMENTS}

This research was supported by a grant from the Patient-Centered Clinical Research Coordinating Center funded by the Ministry of Health \& Welfare, Republic of Korea (grant number: HI19C0481, HC19C0103).

The researchers were members of the Multicenter Randomized Controlled Study for Assessing the Usefulness of Suppressing Thyroid Stimulating Hormone Target Levels after Thyroid lobEctomy in Low to Intermediate Risk Thyroid Cancer Patients (MASTER) trial conducted through the Korean Thyroid Association.

\section{ORCID}

Kyungsik Kim https://orcid.org/0000-0001-9007-7025

Mijin Kim https://orcid.org/0000-0002-1538-8859

Bo Hyun Kim https://orcid.org/0000-0001-9632-9457
Sue K. Park https://orcid.org/0000-0001-5002-9707

\section{REFERENCES}

1. Pellegriti G, Frasca F, Regalbuto C, Squatrito S, Vigneri R. Worldwide increasing incidence of thyroid cancer: update on epidemiology and risk factors. J Cancer Epidemiol 2013; 2013:965212.

2. Korea Central Cancer Registry, National Cancer Center, Korea. Annual report of cancer statistics in Korea in 2018 [Internet]. Goyang: National Cancer Center; 2021 [cited 2021 Aug 10]. Available from: https://ncc.re.kr/cancerStatsView. ncc?bbsnum $=558 \&$ searchKey $=$ total $\&$ searchValue $=\&$ page Num $=1$.

3. International Agency for Research on Cancer, World Health Organization. Cancer today, Globocan 2020 [Internet]. Lyon: IARC; 2020 [cited 2021 Aug 10]. Available from: https://gco.iarc.fr/today/home.

4. Ahn HS, Kim HJ, Welch HG. Korea's thyroid-cancer "epidemic": screening and overdiagnosis. N Engl J Med 2014; 371:1765-7.

5. Folland S, Goodman AC, Stano M. The economics of health and health care. New York: Macmillan Publishing Company; 1993.

6. Kwon S. Thirty years of national health insurance in South Korea: lessons for achieving universal health care coverage. Health Policy Plan 2009;24:63-71.

7. Drummond MF, Sculpher MJ, Claxton K, Stoddart GL, Torrance GW. Methods for the economic evaluation of health care programmes. 4th ed. Oxford: Oxford University Press; 2015.

8. Haddix AC, Teutsch SM, Corso PS. Prevention effectiveness: a guide to decision analysis and economic evaluation. 2nd ed. New York: Oxford University Press; 2003. Chapter 8, Cost-effectiveness analysis; p. 156-77.

9. Brown J. Economic evaluation of cancer treatments: a review of the methods. Clin Oncol (R Coll Radiol) 1999;11:78-83.

10. Neumann PJ. Costing and perspective in published cost-effectiveness analysis. Med Care 2009;47(7 Suppl 1):S28-32.

11. Ngorsuraches S. Defining types of economic evaluation. J Med Assoc Thai 2008;91 Suppl 2:S21-7.

12. Vohora D, Singh G. Pharmaceutical medicine and translational clinical research. Boston: Academic Press; 2018. Chapter 33, Pharmacoeconomics in healthcare; p. 465-72.

13. Phillips C, Thompson G. What is cost-effectiveness? Kent: Hayward Medical Communication; 2001.

Copyright (C) 2021 Korean Endocrine Society 
14. McCabe C, Claxton K, Culyer AJ. The NICE cost-effectiveness threshold: what it is and what that means. Pharmacoeconomics 2008;26:733-44.

15. Wang TS, Cheung K, Mehta P, Roman SA, Walker HD, Sosa JA. To stimulate or withdraw?: a cost-utility analysis of recombinant human thyrotropin versus thyroxine withdrawal for radioiodine ablation in patients with low-risk differentiated thyroid cancer in the United States. J Clin Endocrinol Metab 2010;95:1672-80.

16. Donovan PJ, McLeod DS, Little R, Gordon L. Cost-utility analysis comparing radioactive iodine, anti-thyroid drugs and total thyroidectomy for primary treatment of Graves' disease. Eur J Endocrinol 2016;175:595-603.

17. Al-Qurayshi Z, Farag M, Shama MA, Ibraheem K, Randolph GW, Kandil E. Total thyroidectomy versus lobectomy in small nodules suspicious for papillary thyroid cancer: cost-effectiveness analysis. Laryngoscope 2020;130:2922-6.

18. Bergmo TS. How to measure costs and benefits of ehealth interventions: an overview of methods and frameworks. J Med Internet Res 2015;17:e254.

19. Transportation Benefit-Cost Analysis. Benefit-cost ratio [Internet]. Transportation Benefit-Cost Analysis; 2021 [cited 2021 Aug 10]. Available from: http://bca.transportationeconomics. org/types-of-measures/benefit-cost-ratio.

20. Sculpher MJ, Price M. Measuring costs and consequences in economic evaluation in asthma. Respir Med 2003;97: 508-20.

21. Yousefi M, Assari Arani A, Sahabi B, Kazemnejad A, Fazaeli S. Household health costs: direct, indirect and intangible. Iran J Public Health 2014;43:202-9.

22. Jo C. Cost-of-illness studies: concepts, scopes, and methods. Clin Mol Hepatol 2014;20:327-37.

23. Spinks J. Economic considerations of complementary and alternative medicine (CAM) use in Australia [dissertation]. Clayton: Monash University; 2017. https://doi.org/10.4225/ 03/58b3a70ad11a7.

24. National Library of Medicine. National Information Center on Health Services Research and Health Care Technology (NICHSR): Health Economics Information Resources: A Self-Study Course [Internet]. Bethesda: National Library of Medicine; 2016 [cited 2021 Aug 10]. Available from: https:// www.nlm.nih.gov/nichsr/edu/healthecon/04_he_07.html.

25. Prieto L, Sacristan JA. Problems and solutions in calculating quality-adjusted life years (QALYs). Health Qual Life Outcomes 2003;1:80.

26. Lang $\mathrm{BH}$, Wong $\mathrm{CK}$. A cost-minimization analysis compar- ing total thyroidectomy alone and total thyroidectomy with prophylactic central neck dissection in clinically nodal-negative papillary thyroid carcinoma. Ann Surg Oncol 2014;21: 416-25.

27. Shrime MG, Goldstein DP, Seaberg RM, Sawka AM, Rotstein L, Freeman JL, et al. Cost-effective management of low-risk papillary thyroid carcinoma. Arch Otolaryngol Head Neck Surg 2007;133:1245-53.

28. Guo K, Zheng X, Li D, Wu Y, Ji Q, Wang Z. Cost-effectiveness analysis in papillary thyroid carcinoma patients with different neck dissection strategy: a retrospective cohort study. Int J Surg 2018;50:1-5.

29. Walgama E, Randolph GW, Lewis C, Tolley N, Sacks W, Chen Y, et al. Cost-effectiveness of fiberoptic laryngoscopy prior to total thyroidectomy for low-risk thyroid cancer patients. Head Neck 2020;42:2593-601.

30. Kuo EJ, Wu JX, Zanocco KA. Cost effectiveness of immediate biopsy versus surveillance of intermediate-suspicion thyroid nodules. Surgery 2018;164:1330-5.

31. Lang BH, Wong $\mathrm{CK}$, Chan $\mathrm{CT}$. Initial attributable cost and economic burden of clinically-relevant differentiated thyroid cancer: a health care service provider perspective. Eur J Surg Oncol 2015;41:758-65.

32. Kim SY, Kim SM, Chang H, Kim BW, Lee YS, Kwon SS, et al. Cost for treatment and follow-up of thyroid cancer increases according to the severity of disease. Head Neck 2019;41:2376-9.

33. Lin JF, Jonker PK, Cunich M, Sidhu SB, Delbridge LW, Glover AR, et al. Surgery alone for papillary thyroid microcarcinoma is less costly and more effective than long term active surveillance. Surgery 2020;167:110-6.

34. Oda H, Miyauchi A, Ito Y, Sasai H, Masuoka H, Yabuta T, et al. Comparison of the costs of active surveillance and immediate surgery in the management of low-risk papillary microcarcinoma of the thyroid. Endocr J 2017;64:59-64.

35. Boltz MM, Hollenbeak CS, Schaefer E, Goldenberg D, Saunders BD. Attributable costs of differentiated thyroid cancer in the elderly Medicare population. Surgery 2013; 154:1363-70.

36. Lubitz CC, Kong CY, McMahon PM, Daniels GH, Chen Y, Economopoulos KP, et al. Annual financial impact of welldifferentiated thyroid cancer care in the United States. Cancer 2014;120:1345-52.

37. Lang BH, Wong CK. A cost-effectiveness comparison between early surgery and non-surgical approach for incidental papillary thyroid microcarcinoma. Eur J Endocrinol 
2015;173:367-75.

38. Lee L, How J, Tabah RJ, Mitmaker EJ. Cost-effectiveness of molecular testing for thyroid nodules with atypia of undetermined significance cytology. J Clin Endocrinol Metab 2014;99:2674-82.

39. Wilson L, Huang W, Chen L, Ting J, Cao V. Cost effectiveness of lenvatinib, sorafenib and placebo in treatment of radioiodine-refractory differentiated thyroid cancer. Thyroid 2017;27:1043-52.

40. Venkatesh S, Pasternak JD, Beninato T, Drake FT, Kluijfhout WP, Liu C, et al. Cost-effectiveness of active surveillance versus hemithyroidectomy for micropapillary thyroid cancer. Surgery 2017;161:116-26.

41. Zanocco K, Heller M, Elaraj D, Sturgeon C. Cost effectiveness of intraoperative pathology examination during diagnostic hemithyroidectomy for unilateral follicular thyroid neoplasms. J Am Coll Surg 2013;217:702-10.

42. Cleemput I, Neyt M, Thiry N, De Laet C, Leys M. Threshold values for cost-effectiveness in health care. Belgian Health Care Knowledge Centre. HTA reports. Brussels: KCE; 2008. p. 88.
43. Lohia S, Hanson M, Tuttle RM, Morris LG. Active surveillance for patients with very low-risk thyroid cancer. Laryngoscope Investig Otolaryngol 2020;5:175-82.

44. Haugen BR, Alexander EK, Bible KC, Doherty GM, Mandel SJ, Nikiforov YE, et al. 2015 American Thyroid Association management guidelines for adult patients with thyroid nodules and differentiated thyroid cancer: the American Thyroid Association Guidelines Task Force on thyroid nodules and differentiated thyroid cancer. Thyroid 2016;26:1133.

45. Moon JH, Kim JH, Lee EK, Lee KE, Kong SH, Kim YK, et al. Study protocol of multicenter prospective cohort study of active surveillance on papillary thyroid microcarcinoma (MAeSTro). Endocrinol Metab (Seoul) 2018;33:278-86.

46. Lee EK, Kang YE, Park YJ, Koo BS, Chung KW, Ku EJ, et al. A multicenter, randomized, controlled trial for assessing the usefulness of suppressing thyroid stimulating hormone target levels after thyroid lobectomy in low to intermediate risk thyroid cancer patients (MASTER): a study protocol. Endocrinol Metab (Seoul) 2021;36:574-81. 